\title{
Spektroskopische Untersuchungen an elektrischen Entladungen in Strahlen aus kondensiertem Wasserstoff
}

\author{
R. KLINGELHöFER, H. RöHL und J. Wüst \\ Institut für Kernverfahrenstechnik der Technischen Hochschule \\ und des Kernforschungszentrums Karlsruhe
}

(Z. Naturforschg. 21 a, 1967-1970 [1966] ; eingegangen am 23. Juli 1966)

\begin{abstract}
Das Emissionsspektrum eines mit Wasserstoff-cluster-Strahlen erzeugten Plasmas wird längs der Strahlrichtung untersucht. Bei einem Elektrodenabstand von $26 \mathrm{~cm}$ wird in einem Bereich von $14 \mathrm{~cm}$ nur die Emission des Wasserstoffs beobachtet, in Elektrodennähe treten auch Linien des Elektrodenmaterials auf.
\end{abstract}

Durch Expansion von Gasen aus einer vorgekühlten Düse und anschließende Überführung des Strahlkerns ins Hochvakuum lassen sich räumlich scharf begrenzte Strahlen aus kondensierter Materie (,cluster"-Strahlen) herstellen ${ }^{1-3}$. Wir haben kürzlich gezeigt, wie sich längs eines cluster-Strahls eine Entladung zünden läßt ${ }^{4-6}$. Dabei entsteht ein Plasma, das nur mit den Elektroden, nicht aber mit den Wänden des Entladungsgefäßes Kontakt hat. Solche Plasmen sind wegen ihrer räumlichen Konzentrierung und der zu erwartenden Reinheit für Kernfusionsexperimente von Interesse.

In der vorliegenden Arbeit wird das Emissionsspektrum eines mit Wasserstoff-cluster-Strahlen erzeugten Plasmas mit einem Quarz-Spektrographen in Abhängigkeit von der Raumkoordinate längs des cluster-Strahls untersucht. Es zeigt sich, daß zwischen zwei 26 Zentimeter voneinander entfernten Elektroden auf einer Länge von 14 Zentimetern der Plasmasäule nur die Linien des Wasserstoffs zu beobachten sind. In der Nähe der Elektroden treten erwartungsgemäß auch Linien des Elektrodenmaterials auf. Jedoch ist die pro Längeneinheit der Plasmasäule vom Wasserstoff abgestrahlte Energie im untersuchten Wellenlängenbereich auch in Elektrodennähe größer als die der Linien des Elektrodenmaterials.

\section{Erzeugung elektrischer Entladungen in Wasserstoff-,,cluster"-Strahlen}

Bei den vorliegenden Versuchen wurden clusterStrahlimpulse mit einer Anordnung erzeugt, die

1 E. W. Becker, K. Bier u. W. Henkes, Z. Phys. 146, 33 [1956].

2 E. W. Becker, R. Klingelhöfer u. P. Lohse, Z. Naturforschg. 15 a, 644 [1960].

3 E. W. Becker, R. Klingelhöfer u. P. Lohse, Z. Naturforschg. 17 a, 432 [1962]. einer früher beschriebenen ähnlich ist ${ }^{3}$. Als Strahlgas dient Wasserstoff mit einem Reinheitsgrad von 99,9\%. Zur Kühlung der Düse wird Wasserstoff in flüssigem Zustand in den Kryostaten eingefüllt und im Unterschied zu den früheren Versuchen durch Verdampfung im abgesperrten Kryostaten auf überkritischen Druck gebracht. Diese Maßnahme gestattet es, zur Steigerung der Molekeldichte im clusterStrahl den Einlaßdruck des Strahlgases ohne Begrenzung durch den Druck im Kryostaten zu erhöhen, weil sich bei überkritischem Druck vor der mit einem Schnellschlußventil verschlossenen Düse keine störende Flüssigkeitsoberfläche ausbilden kann. Der Raum zwischen der Düse und dem Strahlabschäler wird durch eine Roots-Pumpe von $250 \mathrm{~m}^{3} / \mathrm{h}$ Saugvermögen evakuiert. An den Raum zwischen dem Abschäler und dem Kollimator ist nur eine Öl-Dampfstrahlpumpe von $250 \mathrm{l} / \mathrm{sec}$ und an den Hochvakuumraum eine Öl-Diffusionspumpe von 2000 l/sec Saugvermögen angeschlossen.

Bei allen Versuchen wurde der Kryostatendruck auf 16 atü und der Einlaßdruck des Strahlgases konstant auf 20 atü geregelt. Die Molekeldichte des leicht divergierenden Strahls beträgt nach vorläufigen orientierenden Messungen in der Mitte zwischen den beiden Hochspannungselektroden etwa $5 \cdot 10^{16}$ $\mathrm{H}_{2}$-Molekeln pro $\mathrm{cm}^{3}$. Durch periodisches Auftasten des Schnellschlußventils wird nach jeweils etwa 10 Sekunden ein cluster-Strahlimpuls von einigen Millisekunden Dauer erzeugt. Die Zündung der Entladung durch Elektroneninjektion in den clusterStrahl wurde bereits früher beschrieben ${ }^{4-6}$. Die

${ }^{4}$ E. W. Becker u. R. Klingelhöfer, Z. Naturforschg. 19 a, 813 [1964].

5 E. W. Becker u. R. Klingelhöfer, J. Nucl. Energy, Part C, 8, 413 [1966].

6 R. Klingelhöfer, Beitr. Plasmaphysik 6, 253 [1966]. 
Masse-Elektrode und die Anode (Abb. 1) sind mit einem Kondensator von $0,1 \mu \mathrm{F}$ verbunden, der bei allen Versuchen auf $20 \mathrm{kV}$ geladen wurde. Im Interesse niederinduktiver und möglichst symmetrischer Leitungsführung wird der Entladungsstrom, unterschiedlich zu den früheren Versuchen, von der Masse-Elektrode über zwei breite, parallel zum

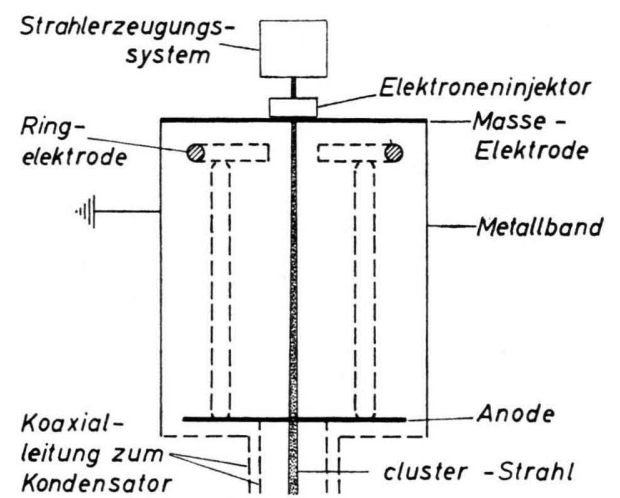

Abb. 1. Anordnung zur Erzeugung elektrischer Entladungen in Wasserstoff-cluster-Strahlen. - Die Koaxialleitung liegt außerhalb der Symmetrieachse des Elektrodensystems, räumlich getrennt vom cluster-Strahl.

cluster-Strahl geführte Metallbänder zum Kondensator zurückgeleitet. Unterhalb der Anode ist der Impulskondensator über eine Koaxialleitung außerhalb der Vakuumkammer angekoppelt. Die teilweise $\mathrm{Ab}$ schirmung des elektrischen Feldes vor der MasseElektrode durch die Metallbänder bedingte eine Vergrößerung der Zündverzugszeit. Zur Vermeidung dieses Effekts ist in $20 \mathrm{~mm}$ Abstand von der MasseElektrode eine ringförmige Elektrode von $180 \mathrm{~mm} \varnothing$ aufgebaut, die von vier auf der Anode befestigten Metallstäben getragen wird. Alle Elektroden sind aus einer Aluminium-Magnesium-Legierung (97\% $\mathrm{Al} ; 3 \% \mathrm{Mg}$ ) hergestellt.

\section{Spektrographie und Auswertung der Meßergebnisse}

Zur spektralen Zerlegung des von der Plasmasäule emittierten Lichtes wurde ein im Bereich zwischen etwa 2000 und $5000 \AA$ verwendbarer QuarzPrismenspektrograph benutzt (Typ „110 M“, Hersteller R. Fu es s, Berlin). Die Spektren wurden mit Ilford-Photoplatten (Typ „Fast Blue Sensitive") aufgenommen. Der optische Strahlengang der Meßanordnung ist aus Abb. 2 ersichtlich. Von einem Teil der leuchtenden Plasmasäule wird ein Zwischen-

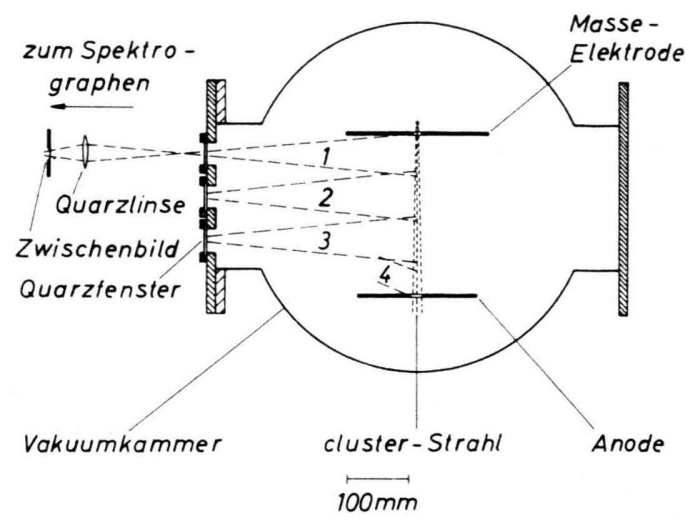

Abb. 2. Anordnung zur Messung des Emissionsspektrums.

bild erzeugt, welches mit einer Kondensorlinse vor dem Eingangsspalt in die Ebene der Kollimatorlinse des Spektrographen abgebildet wird. Durch diese Art der Abbildung wird der Eingangsspalt des Spektrographen von jedem Punkt des zur Abbildung beitragenden Abschnitts der Plasmasäule homogen ausgeleuchtet. Die Linien auf der Photoplatte sind infolgedessen homogen geschwärzt, was für ihre photometrische Auswertung notwendig ist. Durch eine Blende am Ort des Zwischenbildes lassen sich beliebige Teile der Gegenstandsebene, in der die Symmetrieachse des cluster-Strahls liegt, zur spektralen Untersuchung heraussondern. Zur Messung des Emissionsspektrums in Abhängigkeit von der Raumkoordinate längs des cluster-Strahls wurde der Spektrograph in drei verschiedene Positionen gebracht, in denen jeweils ein Abschnitt der Plasmasäule von $60-70 \mathrm{~mm}$ Länge erfaßt wurde. (Abschnitte 1, 2, 3 in Abb. 2.) Der verbleibende Abschnitt 4 vor der Anode konnte aus apparativen Gründen nur unter Schrägaufsicht auf die Anode abgebildet werden. Da bei dieser Abbildung ein anderer Raumwinkel als bei den Abschnitten 1, 2 und 3 erfaßt wird, kann das Meßergebnis von Abschnitt 4 nicht quantitativ mit den anderen Messungen verglichen werden.

Zur Auswertung wurden die Platten photometriert. Aus dem Photometerausschlag erhält man mit Hilfe der Schwärzungskurve der Platten die von dem erfaßten Plasmaabschnitt während der Gesamtzahl der zur Belichtung herangezogenen Entladungen auf die Photoplatte eingeströmte Energie der betreffenden Wellenlänge. Der Photometerausschlag wird durch die Anzahl der zur Belichtung benutzten Entladungen und die Länge des beobachteten Plasmabschnitts 
dividiert, wodurch sich eine auf die Längeneinheit bezogene mittlere Leuchtdichte ergibt. In Abb. 3 ist die Leuchtdichte einiger Linien in Abhängigkeit von der Koordinate längs des cluster-Strahls aufgetra-

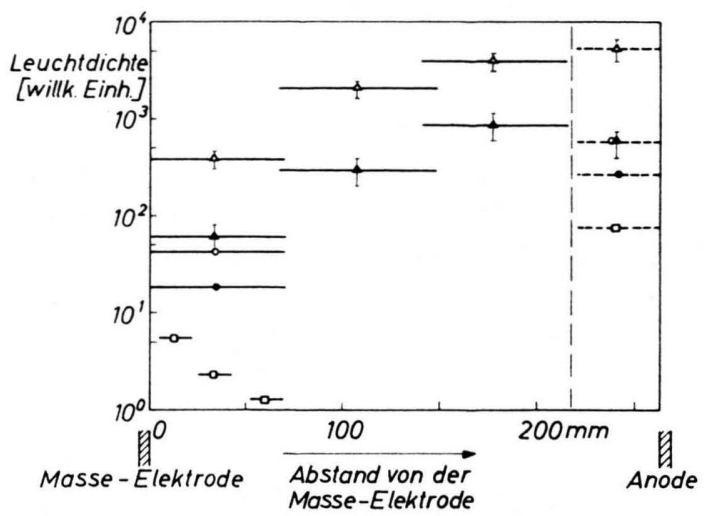

Abb. 3. Ergebnis der photometrischen Auswertung der Spektren in Abhängigkeit von der Koordinate längs des clusterStrahls. - Die Breite der Striche in Abszissenrichtung entspricht der Breite der 4 erfaßten Plasmaabschnitte (s. Abb. 2).

$$
\begin{array}{lllllll}
\triangle & \mathrm{H}_{\beta} & 4861 \AA & \bigcirc & \mathrm{Mg} \text { II } & 2796 \AA \\
\Delta & \mathrm{H}_{\gamma} & 4340 \AA & \bullet & \mathrm{Mg} \text { II } & 2803 \AA \\
& & & & \square & \text { Al II } & 3587 \AA .
\end{array}
$$

gen. Das Ergebnis von Abschnitt 4 ist nur gestrichelt eingezeichnet. Repräsentativ für den Wasserstoff ist nur die Leuchtdichte der intensivsten Linien der Balmer-Serie $\mathrm{H}_{\beta}(4861 \AA)$ und $\mathrm{H}_{\gamma}(4340 \AA)$ eingetragen $\left(\mathrm{H}_{a}\right.$ liegt außerhalb des untersuchten Wellenlängenbereichs) und repräsentativ für die Verunreinigungen die Leuchtdichte der intensivsten $\mathrm{Li}$ nien $\mathrm{Mg}$ II $(2796 \AA), \mathrm{Mg}$ II $(2803 \AA)$ und Al II $(3587 \AA)$. Die Linien der Metallverunreinigungen sind nur in den Abschnitten 1 und 4 der Plasmasäule, nicht aber in den Abschnitten 2 und 3 nachzuweisen. Die Leuchtdichteverteilung der Al-Linie bei $3587 \AA$ wurde repräsentativ für die Linien der Metallverunreinigungen zusätzlich in drei Teilabschnitten innerhalb des Abschnitts 1 mit einem lichtstarken Monochromator photoelektrisch gemessen. Das Ergebnis dieser Messung ist in Abb. 3, gekennzeichnet durch entsprechend kurze Striche in Abszissenrichtung, eingetragen.

In Abschnitt 2, in dem die Leuchtdichte der Wasserstofflinien noch relativ klein ist, waren ungefähr 20 Entladungen notwendig, um die intensivste Linie des Wasserstoffs, die $\mathrm{H}_{\beta}$-Linie $(4861 \AA$ ) aus der Untergrundschwärzung der Platten so stark hervor- treten zu lassen, daß sie photometriert werden konnte. Im Interesse einer möglichst niedrigen Nachweisgrenze für Fremdlinien wurden die Platten mit jeweils 1000 Entladungen belichtet. Damit lag die Nachweisgrenze für eine Linie im Empfindlichkeitsmaximum der Platten $(3500 \AA)$ bei dem ca. $10^{-3}$. fachen der Leuchtdichte der $\mathrm{H}_{\beta}$-Linie im Abschnitt 2 des Plasmas. Wie sich bei den Messungen in Elektrodennähe herausstellte, stehen gerade in diesem Wellenlängenbereich 3 Linien des Elektrodenmaterials als Nachweis für die Verunreinigung des Plasmas zur Verfügung.

Der Kryostat wurde nach jeweils 40 Minuten neu mit flüssigem Wasserstoff gefüllt. Die Messungen wurden immer in einem Zeitintervall zwischen etwa 15 und 40 Minuten nach Einfüllung des flüssigen Wasserstoffs durchgeführt, was jeweils etwa $150 \mathrm{Be}-$ lichtungen der Photoplatten entsprach. In einer gesonderten Messung wurde die maximale Abweichung der gemessenen Leuchtdichte vom Mittelwert während dieses Zeitintervalls zu ungefähr $\pm 30 \%$ ermittelt. Diese Abweichung ist bei der Zusammenstellung der Meßwerte in Abb. 3 in Ordinatenrichtung angegeben.

\section{Ergebnisse und Diskussion der Messungen}

Man entnimmt Abb. 3, daß sich unter den vorliegenden Versuchsbedingungen ein Plasma erzeugen läßt, in dem auf einer Länge von 14 Zentimetern mit der angewendeten spektroskopischen Technik keine Fremdsubstanzen nachzuweisen sind. Das bedeutet, daß in diesem Bereich des Plasmas die Leuchtdichte selbst der intensivsten in Elektrodennähe gefundenen Fremdlinien kleiner ist als das $3 \cdot 10^{-3}$-fache der Leuchtdichte der $\mathrm{H}_{\beta}$-Linie. Die in Abb. 3 dargestellte Abhängigkeit der Leuchtdichte der Aluminium- und Magnesium-Linien zeigt eine Konzentrierung der Metallverunreinigungen in Elektrodennähe. Die Leuchtdichte der intensivsten Magnesium-Linie ist etwa 10-mal kleiner als die der $\mathrm{H}_{\beta}$-Linie. Außerdem handelt es sich bei allen nachgewiesenen Linien des einfach ionisierten Aluminiums und Magnesiums um sogenannte „letzte“ Linien und damit um ein sehr empfindliches Indiz für die Anwesenheit von Elektrodenmaterial im Plasma. Es ist daher anzunehmen, daß die Teilchendichte der Fremdsubstanzen, verglichen mit der Teilchendichte des Wasserstoffs, auch in Elektrodennähe relativ klein ist. 


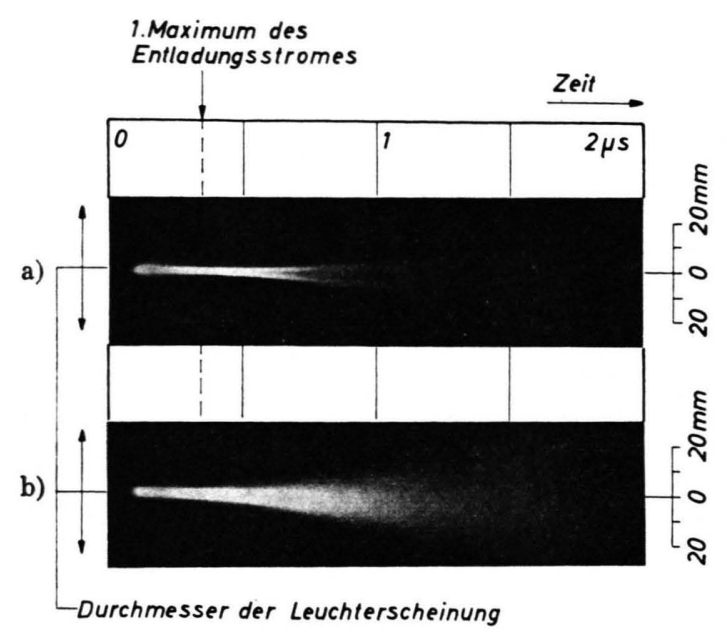

Abb. 4. Schmierbildaufnahmen des Plasmaquerschnitts

a) in $60 \mathrm{~mm}$ Abstand von der Masse-Elektrode,

b) in $180 \mathrm{~mm}$ Abstand von der Masse-Elektrode.

Die Messungen wurden mit einem STL-Bildwandler ausgeführt. Für die Aufnahmen wurde ein $4,5 \mathrm{~mm}$ hoher Querschnitt der Plasmasäule auf die Photokathode des Bildwandlers abgebildet. Die zeitliche Zuordnung von Entladungsstrom und Bildwandleraufnahme ist mit einer Ungenauigkeit von ungefähr \pm 50 nsec behaftet.
Bildwandleraufnahmen, bei denen die Zeitabhängigkeit der Leuchtdichteverteilung eines Plasmaquerschnitts nach der üblichen Schmierbildtechnik aufgenommen wurde, zeigen, daß sich die radiale Struktur der Leuchtdichte längs der Plasmasäule ändert. In der Nähe der Masse-Elektrode ist der Kern der Plasmasäule deutlich dunkler als der Rand (Abb. 4 a). In größerem Abstand von der Masse-Elektrode ist keine ausgeprägte radiale Abhängigkeit der Leuchtdichte wahrnehmbar (Abb. 4 b). Der Effekt kann qualitativ mit der leichten Divergenz des cluster-Strahls und der nur an einem Ende erfolgenden Injektion von Elektronen zur Zündung der Entladung erklärt werden.

Herrn Professor Dr. E. W. Becker danken wir für das Interesse, das er der Arbeit entgegenbrachte, Herrn Dr. O.F. Hagena für die Mitwirkung bei der Konstruktion der Vakuumapparatur und des Hochdruckkryostaten und Fräulein J. Riemenschneider für ihre Hilfe bei den Experimenten.

\title{
Paramagnetische Elektronenresonanz thermisch und durch Strahlung erzeugter Zentren in Bornitrid
}

\author{
G. Römelt
}

Institut für Angewandte Physik der Technischen Hochschule Clausthal

(Z. Naturforschg. 21 a, 1970-1975 [1966] ; eingegangen am 30. August 1966)

By X-rays, ultraviolet, and $\gamma$-radiation or by heating to more than $1850{ }^{\circ} \mathrm{C}$ in powdered or sintered boronnitride centres are produced, which give an EPR-spectrum with 10 lines $a=7.8 \pm 0.1$ Gauss apart with $g=2.0027 \pm 0.0003$ for $\mathrm{BN}$ of natural isotopic composition $\left(81.17 \%{ }^{11} \mathrm{~B} ; 18.83 \%{ }^{10} \mathrm{~B}\right)$, and a single line for ${ }^{10} \mathrm{BN}$ enriched to $91 \%{ }^{10} \mathrm{~B}$. The spectra are analysed as built up from 4 systems of spectra, caused by surroundings of 3 boron atoms, $3,2,1$, or 0 of which are ${ }^{11} \mathrm{~B}$, each occuring with the proper probability. The intensity of the different systems was calculated by assuming Gaussian line shape and equal $|\psi(0)|^{2}$ and line width for ${ }^{11} \mathrm{~B}$ and ${ }^{10} \mathrm{~B}$. It is possible, that the EPR is caused by impurity atoms on the places of $\mathrm{N}$ or in the centre of $\mathrm{B}_{3} \mathrm{~N}_{3}$-rings. Other spectra caused in $\mathrm{BN}$ by X-rays or by neutrons in a pile are discussed elsewhere ${ }^{3,8}$.

Kommerzielles Bornitrid liefert bei der paramagnetischen Elektronenresonanz (EPR) häufig ein 10-Linien-Spektrum (Abb. 1), als dessen Ursache die Hyperfeinwechselwirkung eines Elektrons mit den Kernspins von drei Atomkernen ${ }^{11} \mathrm{~B}$ vermutet wurde $^{1}$, wie sie z. B. in $53,5 \%$ der $\mathrm{B}_{3} \mathrm{~N}_{3}$-Ringe des $\mathrm{BN}$ vorliegen wegen der natürlichen Isotopenhäufigkeit $81,17 \%{ }^{11} \mathrm{~B}$ und $18,83 \%{ }^{10} \mathrm{~B} .{ }^{11} \mathrm{~B}$ hat den Kernspin $I=3 / 2$. Bei dreimaliger $(2 I+1)$-facher Niveau-

1 D. Geist u. G. Römelt, Solid State Commun. 2, 149 [1964]. aufspaltung und $\Delta m_{\mathrm{I}}=0$ bei der EPR ergeben sich zehn Linien.

In Abschnitt I wird eine Analyse des Spektrums durchgeführt, welche die Überlagerung der Einzellinien sowie den Beitrag der ${ }^{10} \mathrm{~B}$ mit $I=3$ berücksichtigt. Unter den Voraussetzungen von Abschnitt I läßt sich dann voraussagen, wie das EPR-Spektrum bei anderer Isotopenzusammensetzung aussehen müßte, z. B. für $91 \%{ }^{10} \mathrm{~B}$, das auch experimentell untersucht wurde. 